\title{
Acute ischemic stroke and COVID-19 pandemic in Brazil: a comparative study of frequency and risk factors before and during SARS-CoV-2 era
}

\author{
Maria Luiza Benevides ${ }^{1,2}$. Stefany Elias ${ }^{3} \cdot$ Pedro Búrigo Costa ${ }^{4} \cdot$ André Luiz Pereira Martins $^{3} \cdot$ Gladys Lentz Martins $^{1}$. \\ Fernando Cini Freitas ${ }^{1} \cdot$ Jean Costa Nunes ${ }^{5,6}{ }^{10}$
}

Received: 27 October 2021 / Accepted: 5 January 2022 / Published online: 9 February 2022

(c) Fondazione Società Italiana di Neurologia 2022

\begin{abstract}
Background COVID-19 pandemic directly impacted the request for hospital care and medical assistance for several diseases worldwide, as occurred with acute ischemic stroke. The present study sought to compare the incidence and severity of acute ischemic stroke (AIS), in addition to sociodemographic, clinical, and radiological characteristics of patients hospitalized in the prepandemic (2018-2019) and pandemic (2020-2021) eras.

Methods An incidence case-control, observational, and analytical research was carried out in the Stroke Unit of Hospital Governador Celso Ramos, Florianopolis, Santa Catarina, Brazil, including 171 patients admitted with acute ischemic stroke from April 2018 to April 2019 (prepandemic era) and 148 patients between January 2020 and January 2021 (during pandemic).

Results The mean incidence of AIS hospital admissions was significantly lower in the pandemic period (CI 95\%, 0.2 to 5.6; $p=0.04$ ), being lower in the lockdown periods and when the incidence of new COVID-19 cases increased. Besides, referring to AIS severity, the mean areas of AIS were larger during the pandemic period $(p<0.01)$, especially in August, September, December, and January $(p<0.05)$. Sociodemographic and clinical variables did not show any difference between the two periods of the study.

Conclusions Hospital admissions for AIS decreased in the COVID-19 pandemic, mostly during months of higher incidences of new COVID-19 cases. When the incidence of admissions diminished, an increase in the severity of AIS was observed, characterized by larger areas. These findings might contribute to other similar referral centers in managing public policies related to stroke.
\end{abstract}

Keywords Acute ischemic stroke $\cdot$ COVID-19 $\cdot$ SARS-CoV-2 $\cdot$ Pandemic

\section{Introduction}

Since the start of the COVID-19 pandemic in December 2019, the impact of the disease on medical services and public health has been profound. Hospitals have worked at

Maria Luiza Benevides and Stefany Elias are joint first authors

Jean Costa Nunes

jeanmedic@hotmail.com

1 Neurology Department, Hospital Governador Celso Ramos (HGCR), Florianópolis, SC, Brazil

2 Neuropediatrics Department, Universidade Estadual de Campinas (UNICAMP), Campinas, SP, Brazil

3 Universidade Do Sul de Santa Catarina (UNISUL), Palhoça, SC, Brazil maximum capacity, large-scale curfews have been established, and isolation and quarantine measures have been introduced [1]. In addition, public health has been challenged to supply services and develop vaccines at the same time [1]. Even tertiary reference centers have been affected and forced to prioritize care for severe coronavirus-related respiratory cases over care for individuals with other serious

4 Internal Medicine Department, Hospital Regional de São José Doutor Homero de Miranda Gomes, São José, SC, Brazil

5 Department of Pathology, Hospital Polydoro Ernani de São Thiago, Universidade Federal de Santa Catarina (UFSC), Professora Maria Flora Pausewang Street, Trindade, SC CEP 88040-000 Florianópolis, Brazil

6 Neurodiagnostic Brasil - Diagnósticos em Neuropatologia, Florianópolis, SC, Brazil 
diseases, such as myocardial infarction (MI) [2] and stroke [3-9]. In addition, public anxiety and fear of the contagious COVID-19 have contributed to self-isolation [1] and mitigated hospital admissions for other diseases [2, 6].

Before the pandemic, the world's acute ischemic stroke (AIS) age-standardized incidence per 100,000 person-years ranged from 51.88 in Qatar to 433.97 in Lithuania [10], with approximately 11 million people diagnosed with stroke each year [10]. Most of the cases of AIS took place in low- and middle-income countries, accounting for $63 \%$ of cases [10]. In Brazil, the latest prepandemic studies showed a stroke incidence rate of $105.4 / 100,000$, of which $80.4 \%$ are due to AIS, and the mortality rate is $23.9 / 100,000$ [11]. Considering the high incidence, prevalence, and burden of AIS, continued studies are warranted to raise strategies to better attend to individuals with the disease during the pandemic, especially considering that the situation is extremely dynamic.

Some studies have investigated reduced admissions and in-hospital delays for treatment delivery and showed the impact of the COVID-19 outbreak on AIS care [3-8]. They demonstrated that stroke treatment has been affected independent of the global region, affecting Europe [3-6], North America [7, 8], and the East [9]. Since the outbreak of COVID-19, studies have shown that acute stroke alerts and hospitalizations decreased [3], patients had more severe baseline deficits [9] and they were worse in ASPECTS on hospital admission [6], intravenous thrombolysis decreased or was delayed [5, 9], primary endovascular procedures increased in some centers [5], and the number of strokes of undetermined origin increased [6]. Therefore, wide coverage of world regions is necessary to clarify the present context. There are few studies in South America that compare acute stroke hospital admissions and care before and since the outbreak of COVID-19 [12], and most foreign studies have short-term comparisons. Brazil recorded its first COVID-19 patient on 25 February 2020, and the state of emergency was declared on 20 March 2020.

This study aimed to compare the epidemiological, clinical, and treatment characteristics of AIS patients admitted to a stroke unit located in southern Brazil during a 1-year period before (from April 2018 to April 2019) and since (from January 2020 to January 2021) the outbreak of COVID-19.

\section{Methods}

This was an incidence case-control study [13] of patients diagnosed with AIS admitted to the Hospital Governador Celso Ramos-Stroke Unit (HGCR-Stroke Unit), a referral center located in southern Brazil. Patients were consecutively evaluated during the pandemic between January
2020 and January 2021; these data were compared with a study before the onset of the COVID-19 pandemic (from April 2018 to April 2019) carried out in the same neurological center that applied the same inclusion criteria.

The study sampling was performed with the census form that evaluated all patients diagnosed with AIS. The included patients were admitted to the HGCR-Stroke Unit, April 2018 to April 2019 and January 2020 to January 2021. Patients were $\geq 20$ years old and had been diagnosed with anterior circulation ischemic stroke, defined by persistent neurological deficits over $24 \mathrm{~h}$ and well-defined lesions detected in neuroimaging exams (cranial tomography, CT, and/or resonance magnetic imaging (MRI)), occurring in the anterior cerebral artery and/or middle cerebral artery vascular territories.

The exclusion criteria were as follows: (1) another diagnosis during hospitalization, including transient ischemic accident (TIA) or hemorrhagic stroke; (2) absence of established lesion in neuroimaging; (3) posterior stroke; (4) hospital discharge, transfer, or evasion before the diagnostic confirmation of ischemic stroke; (5) condition that hindered the patient from understanding and adequately answering the questionnaire, such as previous severe dementia, severe aphasia (global, Wernicke, or sensorial transcortical aphasias), reduced consciousness (Glasgow Coma Scale $\leq 9$ ), confusion, or amnesia; (6) repeated interviews, in case of the second episode of ischemic stroke during each study period; (7) presence of another comorbidity in the central nervous system (CNS), such as central venous thrombosis, previous severe traumatic brain injury, or brain tumors; (8) death in the present hospitalization; (9) and a COVID-19 diagnosis upon hospital admission.

Medical researchers administered a nonvalidated standardized questionnaire to patients included in the study after a training period focused on questionnaire implementation and neurological examination. The questionnaire included data from the patients' medical history, neurological exam, and neuroimaging.

The included demographic and clinical variables were sex, age, vascular hypertension, diabetes mellitus type 2, smoking, and stroke treatment (thrombolysis and/or thrombectomy). The researchers applied the National Institute Health Stroke Scale (NIHSS), and cranial CT and/or MRI was performed 24 to $72 \mathrm{~h}$ after the ictus. Two trained researchers analyzed the neuroimaging exams using the WEASIS Medical Viewer program. The delimitation of the infarction area was estimated in the axial section, considering the largest area of ischemia (measured in square millimeter). Therefore, variables related to stroke neuroimaging were also included, such as hemisphere and vascular territory involvement. Imaging data were revised to identify strokes possibly related to the embryonal variant 
of the posterior cerebral artery, and they did not occur among the sample.

Patients were categorized into two groups, considering the time of HGCR-Stroke Unit admission: (1) patients admitted before the COVID-19 pandemic (2018-2019) and (2) patients admitted during the COVID-19 pandemic (2020-2021). Their clinical, neurological, and radiological data were compared to evaluate the differences in the frequency of hospital admissions and severity of AIS before and during the COVID19 pandemic.

The present study was approved by the Ethics Committee of Hospital Governador Celso Ramos (HGCR) according to the ethical standards of the Code of Ethics of the World Medical Association (Declaration of Helsinki, 1964) and its later amendments (numbers of registration 3.036.226 and 3.794.456). Informed consent was obtained from all patients before the interview.

Statistical analysis was performed using SPSS 23.0 (SPSS Inc., Chicago, IL, USA). Two-line graphics were drafted. The first one compares the monthly incidence of AIS admissions before and during the COVID-19 pandemic in addition to the incidence of new COVID-19 cases in Brazil. Incidences before and during the pandemic were compared month by month. The second graphic compares the mean area of AIS, also monthly and before and during the COVID-19 pandemic. Continuous demographic and clinical variables were expressed by means and standard deviations (SDs). Categorical clinical variables were described using proportions and percentages. Initially, we investigated the differences in demographic, clinical, and radiological variables between the groups (before and during the COVID-19 pandemic) with the chi-square test for categorical variables, Student's $t$-test for continuous parametric variables, and the Mann-Whitney $U$ test for nonparametric variables. The severity of stroke was established by NIHSS and the stroke area $\left(\mathrm{mm}^{2}\right)$. The difference in NIHSS and stroke area between the groups (before and during the COVID-19 pandemic) was defined using the Mann-Whitney $U$ test and Student's $t$-test, after normality tests (Kolmogorov-Smirnov and Shapiro-Wilk). The magnitude of the difference between the groups was measured by the crude odds ratio (OR) and 95\% confidence intervals (CI 95\%). $p$ levels lower than 0.05 were considered significant. The mean area and NIHSS scores of AIS were stratified into three trimester categories, which included the overlapping months between the two periods (April 2018 to January 2019 compared to April 2020 to January 2021), and the difference between them was verified using ANOVA.

\section{Results}

From the total number of 374 AIS patients admitted before (2018-2019) and during (2020-2021) the pandemic, according to the inclusion and exclusion criteria, a total of 319 patients were included in the study (171 before and 148 during the pandemic). Therefore, we interviewed 171 patients with AIS admitted to the HGCR in the prepandemic period between April 2018 and April 2019. In the pandemic period, 148 patients were interviewed between January 2020 and January 2021.

Among the patients interviewed in the prepandemic period, $101(59.1 \%)$ were male, and the mean age was 64.5 years $(S D \pm 12.9)$. Regarding the clinical variables, $134(78.4 \%)$ had a previous diagnosis of hypertension, 55 (32.2\%) had diabetes, and 50 (29.2\%) were active smokers. The mean NIHSS score was $4.7(S D \pm 4.1)$, and the mean stroke area was $707 \mathrm{~mm}^{2}(S D \pm 940.4)$. Thirteen $(7.6 \%)$ patients underwent thrombolysis, and 11 (6.4\%) had hemorrhagic transformation proven by imaging exam (CT or MRI). The mean time of hospitalization was 10.7 days $(S D \pm 11.8)$.

On the other hand, considering the patients evaluated in the pandemic period, $79(53.4 \%)$ were male, and the mean age was 64.5 years $(S D \pm 13.9)$. Clinical variables showed 105 (70.9\%) patients had hypertension, 45 (30.4\%) had diabetes, and 38 (25.7\%) were active smokers. Furthermore, the mean NIHSS score was 4.25 ( $S D \pm 4.3)$, and the mean stroke area was $921.6 \mathrm{~mm}^{2}(S D \pm 1039.1)$. Twenty-three (15.5\%) patients underwent thrombolysis, and 22 (14.9\%) had hemorrhagic transformation. The mean time of hospitalization was 10.5 days $(S D \pm 6.7)$. Table 1 describes these data.

An association between thrombolysis and the pandemic period was detected (OR 2.2; CI 95\% 1.1 to $4.6 ; p=0.03$ ), and an association between hemorrhagic transformation and the pandemic period was also detected (OR 2.5; CI $95 \% 1.2$ to $5.4 ; p=0.02$ ). The means of ischemic areas were significantly different comparing both categories, being superior in the pandemic period $(p<0.01)$. Sociodemographic (sex and age) and clinical (hypertension, diabetes, current smoking, NIHSS, and time of hospitalization) variables did not show any difference between the two periods of the study. Table 1 shows the clinical characteristics of the participants in both periods.

Table 2 shows the differences between the stroke areas according to the prepandemic and pandemic periods, stratified in three trimesters of each evaluated period. There was a difference between the mean of the areas in the second trimester, with larger areas in the pandemic period evaluated by the Mann-Whitney $U$ test $(p=0.04)$. The final statistical analysis, using ANOVA, showed no significant difference in the means of the areas or NIHSS scores between the three trimesters of the pandemic period. 
Table 1 Sociodemographic and clinical differences among ischemic stroke patients admitted to the hospital before (2018-2019) and during (2020-2021) the COVID-19 pandemic

\begin{tabular}{|c|c|c|c|c|}
\hline Categorical variables & $\begin{array}{l}\text { Pre-pandemic } \\
\quad(n=171), N \\
(\%)\end{array}$ & $\begin{array}{l}\text { During pandemic } \\
\quad(n=148), N(\%)\end{array}$ & Odds ratio (CI 95\%) & $p$ value \\
\hline \multicolumn{5}{|l|}{ Sex } \\
\hline Female & $70(40.9)$ & $69(46.6)$ & 1 & \multirow[t]{2}{*}{0.31} \\
\hline Male & $101(59.1)$ & $79(53.4)$ & $0.8(0.5-1.2)$ & \\
\hline \multicolumn{5}{|l|}{ Hypertension } \\
\hline No & 37 (21.6) & $43(29.1)$ & 1 & \multirow[t]{2}{*}{0.15} \\
\hline Yes & $134(78.4)$ & $105(70.9)$ & $0.7(0.4-1.1)$ & \\
\hline \multicolumn{5}{|l|}{ Diabetes } \\
\hline No & $116(67.8)$ & $103(69.6)$ & 1 & \multirow[t]{2}{*}{0.81} \\
\hline Yes & $55(32.2)$ & $45(30.4)$ & $0.9(0.6-1.5)$ & \\
\hline \multicolumn{5}{|l|}{ Current smoke } \\
\hline No & $121(70.8)$ & $110(74.3)$ & 1 & \multirow[t]{2}{*}{0.53} \\
\hline Yes & $50(29.2)$ & $38(25.7)$ & $0.8(0.5-1.4)$ & \\
\hline \multicolumn{5}{|l|}{ Thrombolysis } \\
\hline No & $158(92.4)$ & $125(84.5)$ & 1 & \multirow[t]{2}{*}{0.03} \\
\hline Yes & $13(7.6)$ & $23(15.5)$ & $2.2(1.1-4.6)$ & \\
\hline \multicolumn{5}{|l|}{ Hemorrhagic transformation } \\
\hline No & $160(93.6)$ & $126(85.1)$ & 1 & \multirow[t]{2}{*}{0.02} \\
\hline Yes & $11(6.4)$ & $22(14.9)$ & $2.5(1.2-5.4)$ & \\
\hline Continuous variables & Mean $(\boldsymbol{S D})$ & Mean $(S D)$ & & \\
\hline Age (years)* & $64.5( \pm 12.9)$ & $64.5( \pm 13.9)$ & N.A & 0.89 \\
\hline NIHSS* & $4.7( \pm 4.1)$ & $4.25( \pm 4.3)$ & N.A & 0.11 \\
\hline Stroke area $\left(\mathrm{mm}^{2}\right)^{*}$ & $707( \pm 940.4)$ & $921.6( \pm 1,039.1)$ & N.A & $<0.01$ \\
\hline Time of hospitalization (days)* & $10.7( \pm 11.8)$ & $10.5( \pm 6.7)$ & N.A & 0.10 \\
\hline
\end{tabular}

N.A. not applied, NIHSS National Institute of Health Stroke Scale

*Mann-Whitney $U$ test was applied

Table 2 Mean ischemic stroke areas in different trimesters of patients admitted to the hospital before (2018-2019) and during (2020-2021) the COVID-19 pandemic

\begin{tabular}{llll}
\hline Stroke areas $\left(\mathrm{mm}^{2}\right)^{*}$ & Pre-pandemic, mean $(\mathrm{SD})$ & During pandemic, mean $(\mathrm{SD})$ & $p$ value \\
\hline First trimester & $697.2( \pm 974.4)$ & $677.6( \pm 762.2)$ & 0.51 \\
Second trimester & $707.3( \pm 838.1)$ & $1,281.2( \pm 1404.8)$ & 0.04 \\
Third trimester & $715.3( \pm 968.4)$ & $944.5( \pm 1079.2)$ & 0.13 \\
\hline
\end{tabular}

*Mann-Whitney $U$ test was applied

Figure 1 represents the incidence of hospitalizations for AIS in the HGCR from April 2018 to January 2019 compared to the pandemic period from April 2020 to January 2021. There is also a representation of new cases COVID19 cases in Brazil from April 2020 to January 2021. In the graphic, we can observe that the incidence of hospitalizations is lower in April 2020 than in 2018, similar to the incidence of hospitalizations in January 2021 compared to January 2018. The mean incidence of AIS admissions was significantly lower in the pandemic period ( 11.3 cases per month; $S D \pm 2.9)$ than in the prepandemic period (14.2 cases per month; $S D \pm 3.6$ ) (CI 95\% 0.2 to $5.6 ; p=0.04$ ).
Figure 2 represents the mean areas of AIS in each month between April 2018 and January 2019 (prepandemic) compared with each month between April 2020 and January 2021 (during pandemic). There was a statistically significant difference between the mean areas of AIS in August, September, December, and January, with larger areas during the pandemic period. 
Fig. 1 Graphic representation of the incidence rate of hospitalizations of ischemic stroke between April 2018 and January 2019 (light gray line) and between April 2020 and January 2021 (black line). The blue line represents the incidence of new cases of COVID-19 in the same period, in Brazil

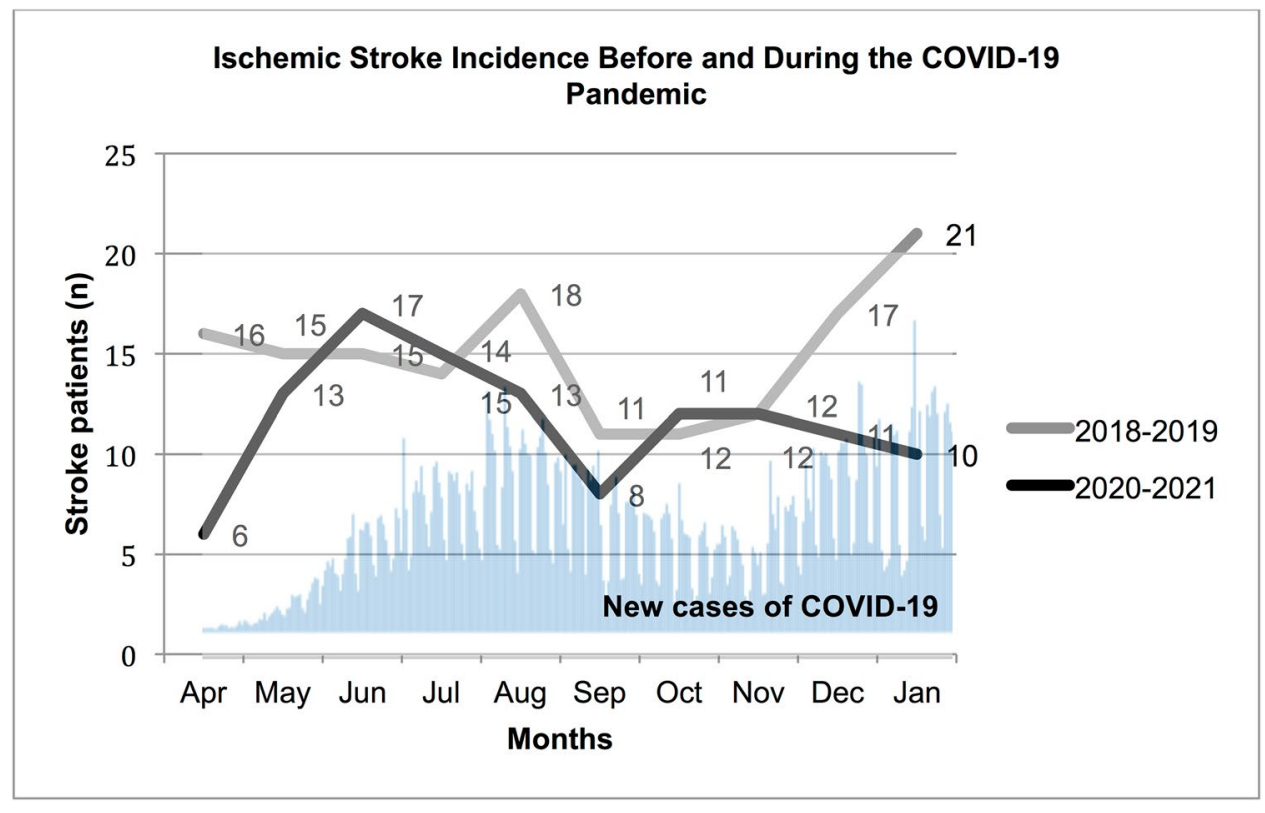

Fig. 2 Graphic representation of the mean areas of ischemic strokes between April 2018 and January 2019 (light gray line) and between April 2020 and January 2021 (black line). $* p<0.05$

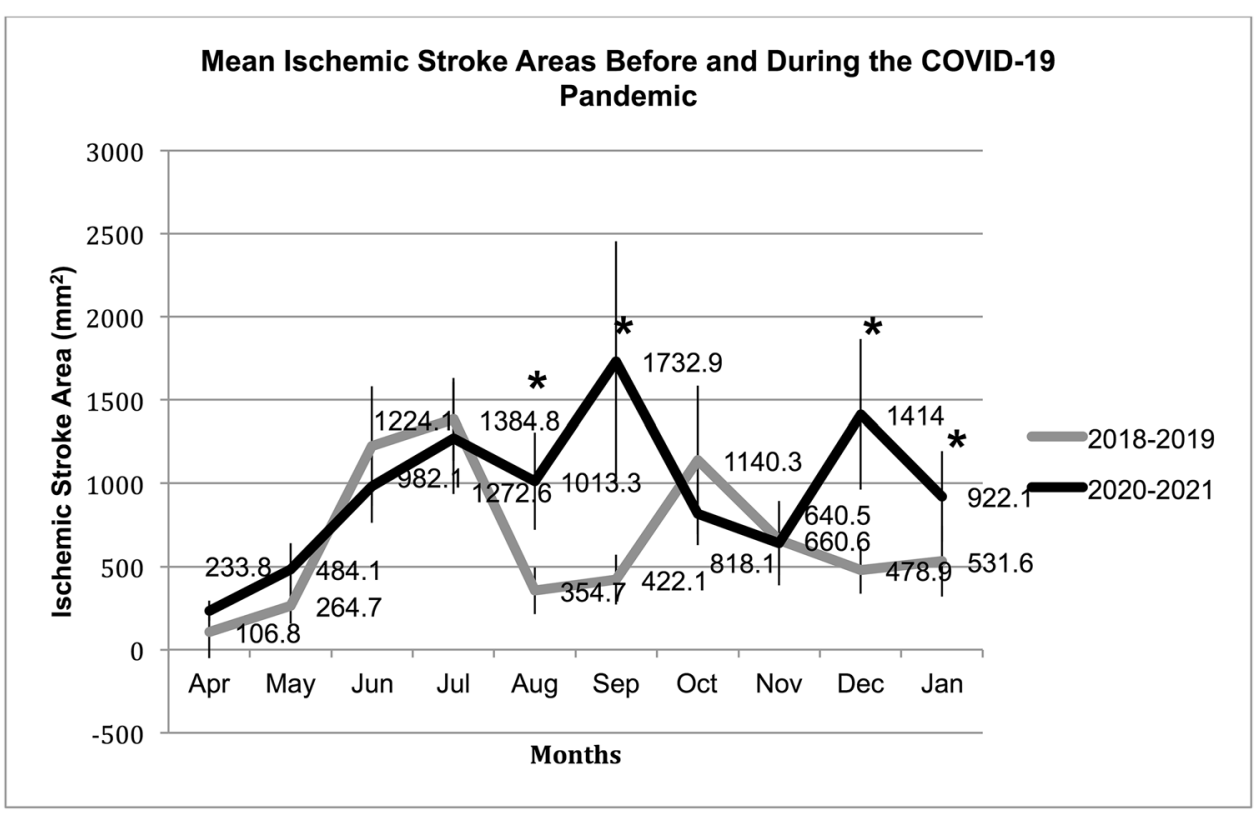

\section{Discussion}

The present study showed that during the pandemic, the mean incidence of AIS hospitalizations per month significantly decreased, especially in the periods following an increase in new COVID-19 cases. A marked decline in AIS admissions was seen during the lockdown in southern Brazil in April, and after a few months, the admissions became similar to the prepandemic period. In addition, the highest COVID-19 incidences did not match the AIS incidence peaks.
Previous data might explain the oscillations in AIS incidence observed in our study. Global anxiety and fear of COVID-19 infection could have led to self-isolation, and forced isolation during lockdown periods was required [1], which could have reduced hospital admissions for stroke, as they did for other diseases [14], such as acute MI and trauma $[2,15,16]$. The overall decrease in hospital admissions ranged from 35 to $40 \%$ in the lockdown period [14-16]. On the other hand, we could expect an increase in AIS incidence during the pandemic, since stroke is a vascular disease possibly triggered by SARS-CoV-2 infection [17], which did not 
occur in our sample. Despite endothelial dysfunction and the prothrombotic state in COVID-19, the causal relationship between SARS-CoV-2 and stroke remains unproven [17].

Concerning the comparisons between the periods before and during the pandemic, in our sample, we found no differences in the sociodemographic and clinical profiles of the evaluated patients during the two periods, in accordance with similar studies. An Italian study by Sacco et al. evaluated data from patients with stroke in both periods and demonstrated no difference between sex and age [4]. Another study by Erol et al., which sought to compare the biological behavior of MI before and during the pandemic, showed interesting data about comorbidities, such as hypertension and diabetes, and reported the same prevalence when comparing both periods [2].

Despite no differences in previous clinical comorbidities, some changes were noted in AIS treatment, such as a higher incidence of thrombolysis and hemorrhagic transformation during the pandemic period. On the other hand, no difference was seen in the time of hospitalization before and during the pandemic. These were unexpected findings since previous studies showed a decrease or delay of thrombolysis [5, $6,9]$, in addition to an increase in strokes of undetermined origin probably related to briefer hospitalizations [6]. We might raise the possibility that individuals with nondisabling minor strokes stayed at home [6], leading to an increase in the overall thrombolysis indication. In addition, more frequent indications of thrombolysis and larger areas of AIS during the pandemic might have contributed to the higher incidence of hemorrhagic transformations.

Regarding AIS severity, the NIHSS showed no difference when the prepandemic and pandemic eras were compared, in accordance with studies conducted in France, Spain, and Japan [3, 6, 9]. However, an important difference between the mean areas between the two periods was verified in our research, being larger in the pandemic period. These findings suggest that stroke area might be a measure for stroke severity, besides NIHSS. In addition, it highlights that the more severe AIS patients were hospitalized during the pandemic, likely requiring extra effort on the part of hospitals to provide better medical care and multidisciplinary assistance.

The severity of patients looking for medical care before and during the pandemic was studied in other diseases. A study of acute MI conducted in Turkey showed a twofold increase in the risk of major adverse cardiac events, such as heart failure and cardiogenic shock, during the pandemic [2]. The authors attribute this finding, at least partially, to the delay in seeking medical care [18]. In contrast, another study on subarachnoid hemorrhage and traumatic brain injury carried out in Finland showed no difference in the severity of the conditions when comparing January to May 2019 (prepandemic) and the same months in 2020 (during the pandemic period) [18].
A remarkable difference in the mean AIS area was observed comparing the months of August, September, December, and January in the prepandemic and pandemic eras. Larger areas of stroke were detected during the pandemic period, coinciding with moments of higher incidence of COVID-19 cases in Brazil and with the decrement in the incidence of AIS admissions. In April 2020, at the beginning of the lockdown, there was a reduction in the number of hospitalizations for AIS, which was not accompanied by larger areas of stroke, and it may be explained, in part, to the fear of seeking hospital care [1, 14]. However, concerning the periods of December 2020 and January 2021, the reduction in the incidence of hospitalizations is accompanied by statistically larger stroke mean areas, which can be attributed, in part, to the most severely ill patients looking for medical care, as observed in a study on acute coronary syndrome during the COVID19 pandemic [19]. A meta-analysis regarding the influence of seasonal variations on the incidence of AIS showed a higher number of cases in winter [20]. However, meanwhile, the variations of AIS incidence in the pre-pandemic (Fig. 1) and pandemic period in the present study did not follow this pattern.

To better elucidate the period of the pandemic year, in which a greater difference in the means of the stroke areas was noted, stratification by quarters was performed (Table 2). Interestingly, the second trimester of the study (July, August, and September in our sample) showed a significant difference between the mean areas of AIS when comparing the prepandemic and pandemic eras. Few studies have evaluated the evolution of diseases during the pandemic for a year-long period, and few data have compared the severity of the diseases in specific periods of the pandemic.

Some limitations should be acknowledged in interpreting the present study and data. At first, the relatively small sample size might have mitigated the NIHSS differences between the two eras, which was expected considering the marked difference in mean stroke areas. The small sample size might be partially explained by the inclusion and exclusion criteria and informed consent related to the pre-pandemic period. Furthermore, patients with a positive test for COVID-19 were excluded because it is a novel disease, potentially related to stroke, and at the moment, its implications were not fully elucidated. In addition, changes observed in AIS treatment might have been influenced by some structural improvements in the stroke unit during the pandemic, such as increment of neurologists on service, implementation of thrombectomy, and regular clinical meetings to avoid compromise of AIS treatment during this critical period. However, this study also has strong points that might be regarded, such as the 1-year duration of comparison of prepandemic and pandemic eras, very similar populations in each period, and one of the first studies with South American data. 
This study compared AIS data from prepandemic and COVID-19 pandemic periods in a tertiary referral center of neurology. During the pandemic, the incidence of AIS hospitalization significantly decreased, showing oscillations in different moments of this era. A marked decline in AIS cases occurred during the lockdown in southern Brazil and as new COVID-19 cases emerged. In addition, severe AIS, with larger ischemic areas, were observed in the months of highest incidences of new COVID-19 cases in Brazil and of lowest incidences of AIS hospital admissions. Few studies in South America have compared AIS characteristics before and during the COVID-19 pandemic, so this 1-year comparison study helps to clarify the present context of AIS. In addition, it might contribute to other similar referral centers in managing public policies since stroke is one of the most important diseases in the public health context. Future studies are needed to better direct resources and information during the most severe moments of the pandemic.

Acknowledgements We thank the staff from Hospital Governador Celso Ramos for their support in this study.

Author contribution All authors contributed to the study conception and design. Material preparation, data collection, and analysis were performed by BML, ES, CPB, and NJC. The first draft of the manuscript was written by BML and ES, and all authors commented on the previous versions of the manuscript. All authors read and approved the final manuscript.

Data availability The data that support the findings of this study are available from the corresponding author upon reasonable request.

\section{Declarations}

Ethics approval The present study was approved by the Ethics Committee of Hospital Governador Celso Ramos (HGCR) according to the ethical standards of the Code of Ethics of the World Medical Association (Declaration of Helsinki, 1964) and its later amendments (numbers of registration: 3.036.226 and 3.794.456)

Informed consent Informed consent to participate and to publish the study was obtained from all patients before the interview.

Conflict of interest The authors declare no competing interests.

\section{References}

1. Khan M, Adil SF, Alkhathlan HZ, Tahir MN, Saif S, Khan M et al (2020) COVID-19: a global challenge with old history, epidemiology and progress so far. Molecules 26:39

2. Erol MK, Kayıkçıŏlu M, Kılıçkap M, Güler A, Yıldırım A, Kahraman F et al (2020) Treatment delays and in-hospital outcomes in acute myocardial infarction during the COVID-19 pandemic: a nationwide study. Anatol J Cardiol 24:334-342

3. Pop R, Quenardelle V, Hasiu A, Mihoc D, Sellal F, Dugay MH et al (2020) Impact of the COVID-19 outbreak on acute stroke pathways - insights from the Alsace region in France. Eur J Neurol 27:1783-1787
4. Sacco S, Ricci S, Ornello R, Eusebi P, Petraglia L, Toni D, Organization IS (2020) Reduced admissions for cerebrovascular events during COVID-19 outbreak in Italy. Stroke 51:3746-3750

5. Rinkel LA, Prick JCM, Slot RER, Sombroek NMA, Burggraaff J, Groot AE et al (2021) Impact of the COVID-19 outbreak on acute stroke care. J Neurol 268:403-408

6. Tejada Meza H, Lambea Gil Á, Sancho Saldaña A, MartínezZabaleta M, Garmendia Lopetegui E, López-Cancio Martínez E et al (2020) Impact of COVID-19 outbreak in reperfusion therapies of acute ischaemic stroke in northwest Spain. Eur J Neurol 7:2491-2498

7. Siegler JE, Zha AM, Czap AL, Ortega-Gutierrez S, Farooqui M, Liebeskind DS et al (2021) Influence of the COVID-19 pandemic on treatment times for acute ischemic stroke: the Society of Vascular and Interventional Neurology Multicenter Collaboration. Stroke 52:40-47

8. Katsanos AH, de Sa BD, Al-Qarni MA, Shawawrah M, McNicollWhiteman R, Gould L et al (2021) In-hospital delays for acute stroke treatment delivery during the COVID-19 pandemic. Can J Neurol Sci 48:59-65

9. Koge J, Shiozawa M, Toyoda K (2021) Acute stroke care in the with-COVID-19 era: experience at a comprehensive stroke center in Japan. Front Neurol 11:611504

10. Krishnamurthi RV, Feigin VL, Forouzanfar MH, Mensah GA, Connor M, Bennett DA et al (2013) Global and regional burden of firstever ischaemic and haemorrhagic stroke during 1990-2010: findings from the Global Burden of Disease Study 2010. Lancet Glob Health 1:e259-e281

11. Cabral NL, Gonçalves AR, Longo AL, Moro CH, Costa G, Amaral $\mathrm{CH}$ et al (2009) Trends in stroke incidence, mortality and case fatality rates in Joinville, Brazil: 1995-2006. J Neurol Neurosurg Psychiatry 80:749-754

12. Diegoli H, Magalhães PSC, Martins SCO, Moro CHC, França PHC, Safanelli J et al (2020) Decrease in hospital admissions for transient ischemic attack, mild, and moderate stroke during the COVID-19 era. Stroke 51:2315-2321

13. Pearce N (2005) A short introduction to epidemiology. Centre for Public Health Research, Massey University, Wellington, p 130

14. Kapsner LA, Kampf MO, Seuchter SA, Gruendner J, Gulden C, Mate $S$ et al (2021) Reduced rate of inpatient hospital admissions in 18 German university hospitals during the COVID-19 lockdown. Front Public Health 8:594117

15. Mafham MM, Spata E, Goldacre R, Gair D, Curnow P, Bray M et al (2020) COVID-19 pandemic and admission rates for and management of acute coronary syndromes in England. Lancet 396:381-389

16. Wong JSH, Cheung KMC (2020) Impact of COVID-19 on orthopaedic and trauma service: an epidemiological study. J Bone Joint Surg Am 102:e80

17. Nannoni S, de Groot R, Bell S, Markus HS (2021) Stroke in COVID19: a systematic review and meta-analysis. Int J Stroke 16:137-149

18. Luostarinen T, Virta J, Satopää J, Bäcklund M, Kivisaari R, Korja $M$ et al (2020) Intensive care of traumatic brain injury and aneurysmal subarachnoid hemorrhage in Helsinki during the COVID-19 pandemic. Acta Neurochir (Wien) 162:2715-2724

19. De Filippo O, D’Ascenzo F, Angelini F, Bocchino PP, Conrotto F, Saglietto A et al (2020) Reduced rate of hospital admissions for ACS during COVID-19 outbreak in Northern Italy. N Engl J Med 383:88-89

20. Li Y, Zhou Z, Chen N, He L, Zhou M (2019) Seasonal variation in the occurrence of ischemic stroke: a meta-analysis. Environ Geochem Health 41:2113-2130

Publisher's note Springer Nature remains neutral with regard to jurisdictional claims in published maps and institutional affiliations. 